\title{
Direct Laser Writing to Generate Molds for Polymer
}

\section{Nanopillar Replication}

\author{
Colm Delaney,,$^{* *}$ Niamh Geoghegan, ${ }^{1,2}$ Hossam Ibrahim, ${ }^{3,4}$ Mark O'Loughlin, ${ }^{1}$ \\ Brian J. Rodriguez, ${ }^{3,4}$ Larisa Florea, ${ }^{5}$ Susan M. Kelleher ${ }^{1,2}$
}

${ }^{1}$ School of Chemistry, Science Centre - South, University College Dublin, Belfield, Dublin 4, Ireland

${ }^{2}$ CURAM, Science Foundation Ireland Centre for Research in Medical Devices (CURAM), National University of Ireland Galway, Ireland

${ }^{3}$ Conway Institute of Biomolecular and Biomedical Research, University College Dublin, Belfield, Dublin 4, Ireland

${ }^{4}$ School of Physics, University College Dublin, Belfield, Dublin 4, Ireland

${ }^{5}$ School of Chemistry and AMBER, the SFI Research Centre for Advanced Materials and BioEngineering Research, Trinity College Dublin, the University of Dublin, College Green, Dublin 2, Ireland

E-mail: cdelane5@tcd.ie

Keywords: 2-Photon Polymerization, Direct Laser Writing, Replica Molding, Nanopillars, Nanostructured Polymers. 


\begin{abstract}
Herein we exploit direct laser writing as a means to fabricate negative masters for the generation of polymeric nanopillars, via replica molding. Through optimization of design parameters, fabrication configuration, and polymerization protocols, we demonstrate for the first time, the fabrication of large-area negative templates which can be used to repeatedly produce some of the tallest $(>1.5 \mu \mathrm{m})$ and thinnest $(<300 \mathrm{~nm})$ pillars ever achieved in biocompatible photocurable polymers, such as polyethylene glycol diacrylate, polypropylene glycol diacrylate, and polycaprolactone dimethacrylate. This combination serves to add value to two photon polymerization, and to extend its potential to the facile production of millimeter-sized arrays of submicron pillars, of infinite combinations of height, diameter and pitch in a wide variety of compliant, biocompatible, and biodegradable materials.
\end{abstract}

\title{
Introduction
}

The ubiquity of nanostructures in nature, their ability to alter interactions with light and to control hydrophobicity, adhesion, and even cell-interaction is a justification for the breadth of biomimetic nanostructured materials which have been generated over the past twenty years. ${ }^{1-3}$ Inspiration taken from lotus leaves, cicadae, and morpho butterflies has been used to generate significant advances in surface coatings, medicine, and photonics., ${ }^{4,5}$ Synthetic analogues of periodic nanostructured materials have been achieved through disparate approaches, serving as relatively crude approximations for the multifaceted and hierarchical assemblies which are characteristic of natural nanostructures. ${ }^{6}$ In particular, hard lithographic techniques, adapted from the semiconductor industry, such as photolithography, electron beam lithography, and etching have been used to produce high-fidelity nanostructures. ${ }^{7,8}$ To date, these techniques have been hampered 
by high cost, timescale, and the limited nature of the materials used (silicon, titania, glass, polystyrene). ${ }^{9}$ Particular focus on micro/nanofabrication in soft materials has been driven in recent years by advances in photonics, tissue engineering, and medical device research. ${ }^{8-10}$ These advances have focused on primary (hard lithography) techniques, such as electrospinning, e-beam lithography, and direct laser writing, or secondary (soft lithography) techniques such as nanoimprint lithography and capillary micromolding from high-fidelity hard masters, in materials such as silica, anodic alumina, or common negative tone resists. ${ }^{8}$

Of particular interest are direct write techniques, which operate without the need for mask exposure, subsequent photoresist curing, or etching. Laser-based direct write technology using multiple photon absorption can allow spatial confinement of polymerization with minimal topological constraints. Direct Laser Writing (DLW) via 2 photon-polymerization (2PP), in which a laser beam is focused into a tiny volume which serves as a building block within UV-curable photoresists, can yield feature sizes below the diffraction limit of the excitation light. ${ }^{11}$ Since the advent of the technology, research groups have strived to push the boundaries of achievable resolution and line width through innovative physical and chemical approaches. Kawata et al. demonstrated the dramatic effects which could be achieved through the use of additional radical quenchers in the photoresist, which serve to minimize the extent to which created radicals can survive, thereby reducing the effective voxel volume. ${ }^{12}$ Other groups have relied on more traditional methods borrowed from photolithographic process chains, such as post-fabrication baking and shrinkage, to achieve sub $100 \mathrm{~nm}$ line widths. ${ }^{13}$ In recent years, several groups have studied more adaptable acrylate-based photoresists to achieve impressive resolution and accurate predictions of voxel volumes, with respect to laser power and writing speed..$^{14,15}$ 
The flexibility of the 2PP technique, the advancement of throughput in commercially available systems, and the need for arrays of submicron sized structures within optics, photonics, microfluidics, tissue engineering, and cell biology fields, has driven many researchers to make libraries of rapidly prototyped nanostructures in a range of different polymeric materials. Purtov et al. make use of controllable voxel size in proprietary IP-Dip resin to form nanopillars which were constructed from several vertically joined voxels. ${ }^{16}$ Through the use of additional postfabrication crosslinking they have achieved impressive control of shape, pitch, and periodicity, in addition to controllable diameters (from 120 - $430 \mathrm{~nm}$ ) and heights (from $330-1315 \mathrm{~nm}$ ). ${ }^{17}$ The work of the Martinez Group in Copenhagen is also worthy of significant mention for the creation of $250 \mu \mathrm{m} \times 250 \mu \mathrm{m}$ arrays of polymeric nanopillars with submicron diameters, and heights up to $6 \mu \mathrm{m}$, via 2PP, from a pentaerythritol triacrylate monomer. ${ }^{17}$ Their work, which probes the effect of pitch and surface coverage on the ability of fibroblasts to accept cues from the nano- and microtopographies represents a small, but ever-developing, body of research focused on the use of 2PP for biological applications. They demonstrate that without a structural pattern, cells are seen to align randomly, while in the presence of nanopillar geometries, the alignment of cells is observed to be reliant on both pillar height and spacing. To date, much other work surrounding the application in the biological realm has focused on photoresists based on sol-gel chemistry of inorganic-organic hybrid photopolymers, which result in hydrophobic structures that do not swell readily in aqueous media. Koroleva et al. have shown an example of such materials applied within stem cell studies, showing abilities to control seeding efficiency, proliferation, and even differentiation. ${ }^{17}$ Recently, Accardo et al. have shown the use of poly(ethylene glycol) diacrylate with phenylbis(2,4,6-trimethylbenzoyl)phosphine oxide photoinitiator to fabricate 3D architectures on the $10 \mu \mathrm{m}$ feature scale, and have demonstrated their compatibility with cells for 
neuronal tissue engineering. ${ }^{14}$ While some further work has gone on to demonstrate $2 \mathrm{PP}$ fabrication in functionalized gelatin, collagen, and chitosan, with minimal cytotoxicity, adoption of the technology within this field has been rather slow. ${ }^{18}$ Reservations in this regard can arguably be attributed either to a relatively limited library of biocompatible monomers, a paucity of watersoluble photoinitiators with a substantial 2 photon absorption (2PA) cross-section, or indeed, inherent limitations caused by chemical reactivity (susceptibility to Michael reactions), and relatively low conversion rates of common acrylate monomers. Of course, these hurdles have not come without impressive solutions, such as photoinitiators based on coumarin scaffolds with improved water solubility and 2PA cross-sections, ${ }^{19}$ traditional dye-amine combinations which rely on intermolecular electron transfer followed by hydrogen transfer, ${ }^{20}$ and even through the addition of biocompatible surfactants to disperse hydrophobic chromophores. ${ }^{21}$ Nonetheless, a tradeoff between reactivity, biocompatibility, fabrication resolution, and processing throughput may continue to be a sticking-point in extending the application of $2 \mathrm{PP}$. Undeniably, the ability of the technology to produce high-resolution prototypes down to the nanoscale may well find its greatest contribution to biological studies through the combination of complementary techniques.

In this regard, several groups have combined the generation of 2PP-generated templates with more traditional soft lithography techniques on the micron scale, usually through a multistep process which proceeds via a soft, elastomeric replica. Some of the most impressive work in this field has been performed by the group of Helmut Schift at the Paul Scherrer Institute, Villigen, Switzerland, who have drawn on their knowledge of replication to extend it to 2PP generated masters and draw comparisons with silicon templates achieved via dry etch processes. By combining 2PP template generation with supercritical drying, they achieved high aspect ratio photonic nanofences which, via an elastomeric polydimethylsiloxane (PDMS) replica molding step, were then used to generate 
high fidelity replicas of the master in photocurable proprietary-blend polymers. Through intelligent matches of master and replica materials, their results highlight the potential for using such a technique in high-volume manufacturing protocols. ${ }^{22}$ Fourkas et al. have also made significant inroads in this regard over the past fifteen years. Their original work generated structures using 2PP (tens of microns in height and width) in a monomeric cocktail based on 48 wt\% ethoxylated trimethylolpropane triacrylate, $49 \mathrm{wt} \%$ tris(2-hydroxyethyl)isocyanurate triacrylate, and $3 \mathrm{wt} \%$ Lucirin TPO-L. ${ }^{23}$ These structures, with high aspect ratios and truly 3D character, were subsequently used to generate PDMS masters, which could be then used to replicate the original structures in the same cocktail mixtures, without any discernible loss of resolution. The same group has developed on this work, to replicate 3D microstructures with closed loops, and more recently, to replicate submicron size ridges and sawteeth, with widths and heights of several hundred nanometers, via the same two-step process which uses a soft elastomeric primary replica and solvent assisted nanotransfer molding..$^{24,25}$ Despite these significant advances, this approach has yet to be extended to soft, biocompatible polymers, on the nanoscale. Using this method to fabricate large arrays of nanostructures, without the use of any intermediary steps, would allow recipes and formulations currently optimized for bulk polymerization and used across tissue engineering and cell biology, to be accurately and reproducibly nanostructured.

Herein we present a selection of $2.5 \mathrm{D}$ and 3D structures generated from cocktails of biocompatible monomers achieved through the direct replication of 2PP-produced masters. The ability to generate millimeter-sized arrays in soft biocompatible polymers, with the retention of nanostructure, represents a marriage of high-resolution master development with the flexibility of a wellestablished fabrication technology. This approach holds significant potential, as control of material properties such as mechanical strength, degradation, and surface chemistry can be optimized in a 
relatively facile manner. The ability to use $2 \mathrm{PP}$ as a means of generating master templates for the replication of nanoscale grooves, pits, or protrusions, finds great synergy with several decades of work on replication of biocompatible nanotopographies. Moreover, it gives access to highresolution structuring in materials that have not been accessible with $2 \mathrm{PP}$ hitherto.

\section{Results and Discussion}

Arrays of negative masters were fabricated using 2 photon polymerization in photocurable cocktails. The fabrication was performed in the dip-in laser lithography configuration using IPDip photoresist, a proprietary blend containing $60-80 \%$ of 2-(hydroxymethyl)-2-[[(1oxoallyl)oxy]methyl]-1,3-propanediyl diacrylate and supplied by Nanoscribe Gmbh. Covalent attachment of the polymeric structures was achieved by prior silanization of the fused silica substrates. This enabled repeatable replica molding from the same master. Scheme 1 presents a sample fabrication workflow showing replica molding from a 2PP-generated array.

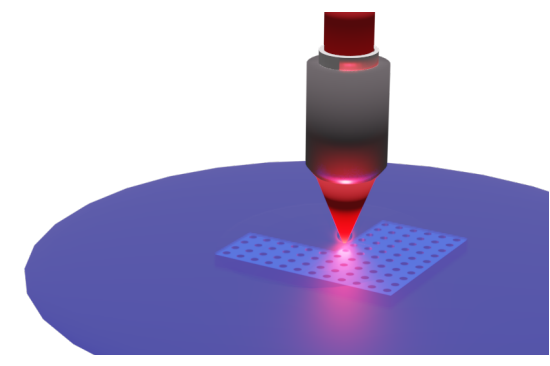

2 Photon Polymerization

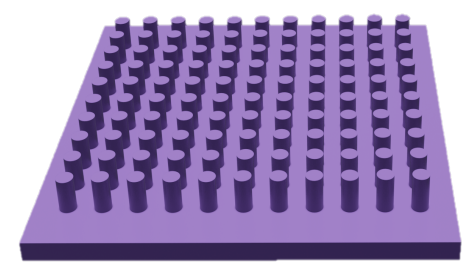

Nanostructured Polymer
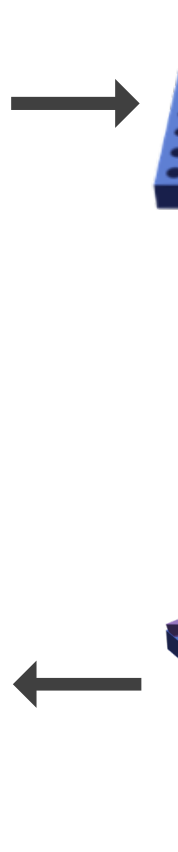

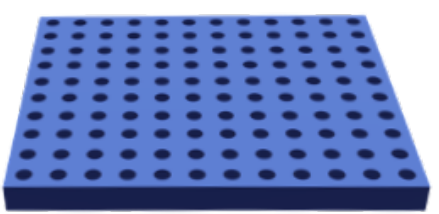

Negative Template

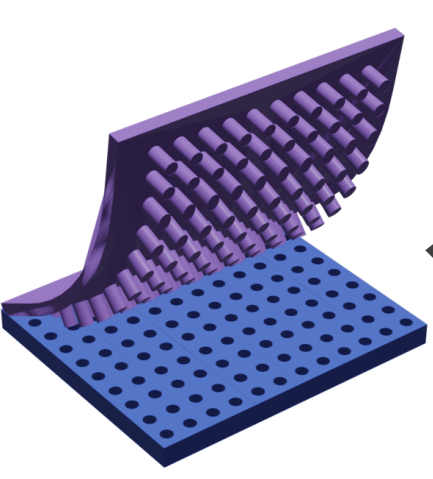

Replica Molding
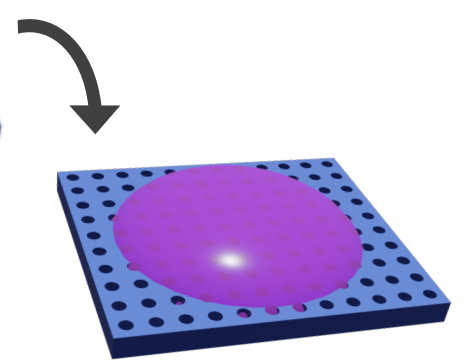

White Light

Polymerization 
Scheme 1: A combination of hard and soft lithography used to generate a library of submicron structured polymers. The process starts with the generation of a negative master using $2 \mathrm{PP}$, followed by replication in photocurable polymers.

Since the advent of 2PP, the effect of writing parameters on feature size and resolution has been widely studied in the context of submicron structures, usually through analysis of adjacent lateral lines or via suspended line structures. Effective voxel size, line width, and polymer growth all play significant roles in the generation of high-resolution submicron features which are constructed by stacking voxels in three dimensions. Much less is known about the effect these parameters have on the generation of high aspect ratio pores, and the resulting size, shape and morphology of the internal channel. Moreover, in the context of replica molding templates for soft photocurable polymers, it is imperative that a systematic study such as this be performed to act as a blueprint for how these two techniques can be brought together to yield structures of predictable and controllable morphology, shape, and size.

To truly understand the effect of fabrication parameters on the generation of high-fidelity replica-molding masters, a single design file of arbitrary dimensions was generated. To create a 3D object from a standard sterolithography (.stl) file using DLW, a solid scaffold is achieved with a series of outline (contour) and filling (hatching) lines. The designs presented herein incorporated a series of $100 \mathrm{~nm}$ slices fabricated on top of each other, with lateral hatching performed perpendicular to the previous layer. This served to negate the effect of fabrication direction on the morphology of the fabricated pore features, which becomes of significant importance at submicron feature sizes. As discussed, fidelity of structures achieved using 2PP, especially in the submicron regime, are a complex product of material, fabrication parameters, and design. To initially probe fabrication parameters for a negative template, when isolated from variables of design, an .stl file 
presented in Figure 1A containing pores of $800 \mathrm{~nm}$ diameter and $800 \mathrm{~nm}$ pitch and of $1.5 \mu \mathrm{m}$ depth was used. In the absence of contouring, which can be used to define the outline of the cylinder, variation of fabrication parameters can have a dramatic effect on the generated pores. The reader is directed to Figure S1 and Table S1 for analysis of the arrays across a range of 20 parameter combinations, varying laser power from $7.5-15 \mathrm{~mW}$ and writing speeds from $1000-8000 \mu \mathrm{ms}^{-}$ 1. All of these parameters yielded pristine free-standing arrays which were fully attached to the fused silica substrate, as demonstrated by the SEM image shown in Figure 1B. Figure 1C presents a representative sample of these arrays, demonstrating, as expected, the profound effect of variation of laser power (increased from $12.5-15 \mathrm{~mW}$ ) on the dimensions of the resulting pores, and the minor effect of varying writing speed through $1000-3000 \mu \mathrm{ms}^{-1}$. The effect of laser power is maximized at slower writing speeds. At laser powers below $10 \mathrm{~mW}$, a significant deviation from circularity was observed. This resulted in pores which tended towards a square geometry with topdown diameters nearing $1 \mu \mathrm{m}$ (such as those fabricated at $7.5 \mathrm{~mW}$ and $1000 \mu \mathrm{ms}^{-1}$; seen in Figure S1). At higher powers (and lower writing speeds) the resulting geometry had an oval shape, as shown in Figure 1, which can be supported by previous studies documenting the effect of power and writing speed on the effective voxel shape and aspect ratio. ${ }^{15}$ Increasing laser power above $17.5 \mathrm{~mW}$ resulted in additional polymerization within the pores, thereby confirming a suitable parameter window for the presented design (Figure S1 and Table S1). 
A

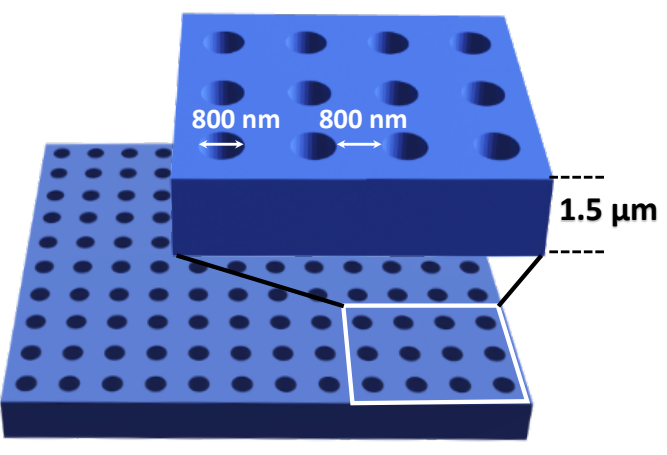

B

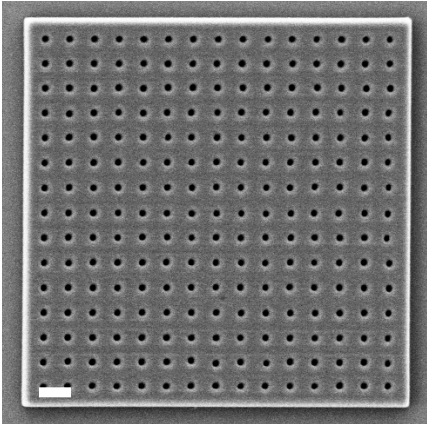

C Increasing Writing Speed

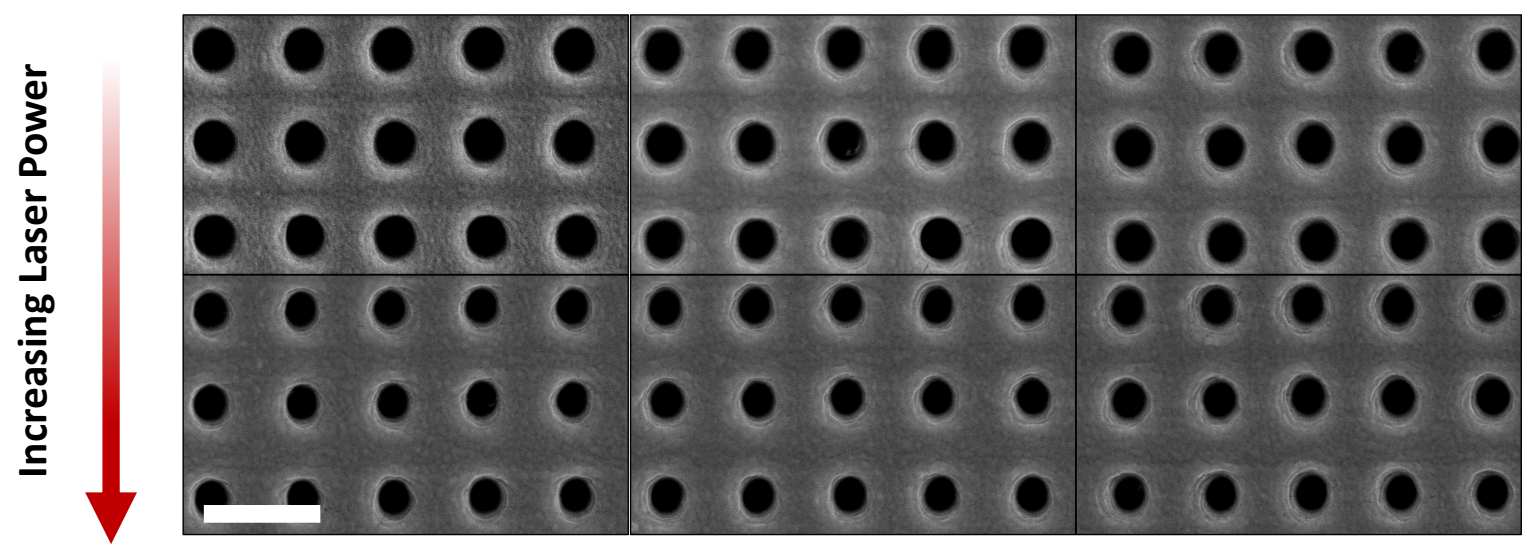

Figure 1: Generation of primary templates; A) .stl design file and B) SEM image of the fabricated master consisting of a $15 \times 15$ pore array. C) SEM images showing the effect of laser power and writing speed on the resulting pores ( $5 \times 3$ pore array shown). Top row fabricated at $12.5 \mathrm{~mW}$ laser power and writing speeds of 1000,2000 , and $3000 \mu \mathrm{ms}^{-1}$, respectively. Bottom row fabricated at $15 \mathrm{~mW}$ laser power and writing speeds of 1000,2000 , and $3000 \mu \mathrm{ms}^{-1}$, respectively. Scale bar represents $2 \mu \mathrm{m}$. All samples were coated with a $10 \mathrm{~nm}$ gold layer to enable imaging and reduce charging.

While top-down analysis of pore diameter proves a useful tool in providing an indication of cylindrical pore size, the true impact of this work is in the generation of $2.5 \mathrm{D}$ and even $3 \mathrm{D}$ objects. Success in template fabrication can only be measured in the ability of these arrays to generate 
replica molded submicron structures, and the height, shape and size thereof. Using a replica molding procedure, outlined in detail in the experimental section, a monomeric cocktail of poly(ethylene glycol) diacrylate $\left(M_{n}\right.$ 575) (PEGDA) with 1 wt\% phenylbis(2,4,6trimethylbenzoyl)phosphine oxide (PBPO) was introduced to the gold-coated (10 nm) masters via capillarity between the substrate slide and a non-silanized top slide, with a channel height of 0.6 mm. Polymerization was then achieved using a white light source, for 120 mins, with irradiation from above and below the sample. A large proportion of the presented arrays successfully replica molded structures in the PEGDA polymer, as outlined in Table S1. For pores created using lower powers/higher writing speeds, the replicated pillars exhibit a notable ellipsoidal geometry, with a bulbous base and tip. This can be observed, in completion, in Figure S2 or a pertinent example (at $12.5 \mathrm{~mW}$ laser power) is observed for pillars replicated from templates fabricated using different writing speeds in Figure 2A, shown in the tilt SEM images. For templates fabricated using writing speeds $<2000 \mu \mathrm{ms}^{-1}$, replicated PEGDA pillars exhibit a traditional cylindrical geometry with uniform diameters from top to bottom, while above speeds of $5000 \mu \mathrm{ms}^{-1}$, the replicated pillars exhibit a hyperboloid geometry with a noticeable overhung plateau at the top. This is particularly pronounced at laser powers of $10 \mathrm{~mW}$ and below, as seen in Figure S2. Conversely, exploiting lower writing speeds, to generate uniform pores in the master template, results in arrays of cylindrical pillars whose diameter can be easily controlled through the laser power used in master fabrication. Two such examples are shown in Figure 2. Tilt SEM images show pillars replicated from pores fabricated at $12.5 \mathrm{~mW}$ power (Figure 2B) with an average diameter of $520 \pm 13 \mathrm{~nm}$ and $15 \mathrm{~mW}$ power (Figure 2C) with an average diameter of $300 \pm 12 \mathrm{~nm}$, respectively, as measured at $60^{\circ}$ tilt. Top down analysis of the primary arrays had diameters of $779 \pm 21 \mathrm{~nm}$ and $726 \pm 22$ $\mathrm{nm}$, respectively. For a designed array with $800 \mathrm{~nm}$ pores, this serves to highlight the profound 
impact writing parameters can have within submicron regimes. Moreover, it further reinforces the assertion that a combination of $2 \mathrm{PP}$ and replica molding can serve to generate potential that is greater than the sum of its constituent parts.
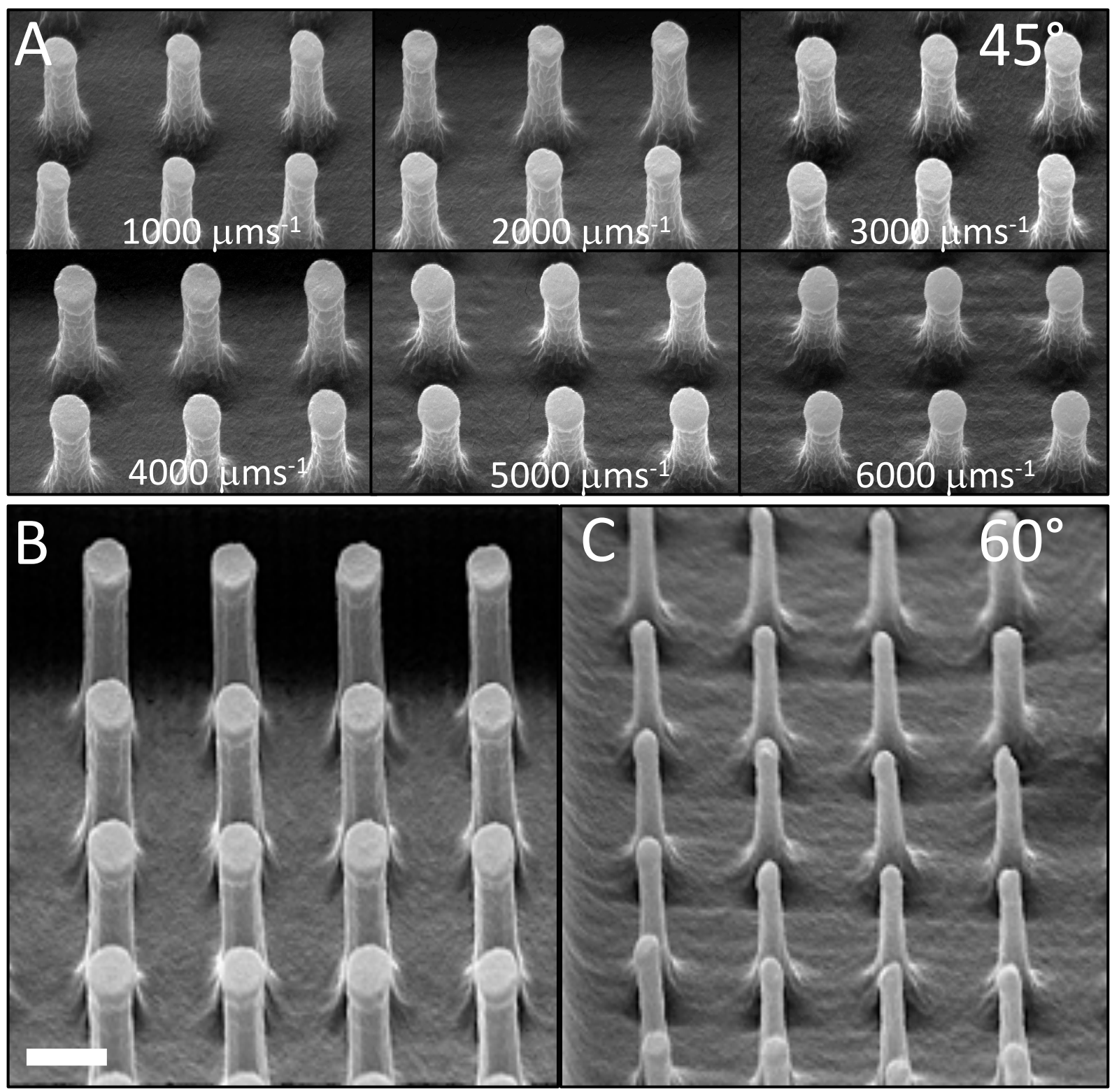

Figure 2: SEM images of PEGDA pillars replicated from 2PP templates. A) shows the effect of writing speed used to form the template on the morphology of resulting pillars $(12.5 \mathrm{~mW}$ laser power, writing speeds from $1000-6000 \mu \mathrm{ms}^{-1}$ ) imaged at $45^{\circ}$ tilt ; B) shows pillars replicated 
from pores fabricated at $12.5 \mathrm{~mW}$ and $2000 \mu \mathrm{ms}^{-1} \mathrm{C}$ ) shows pillars replicated from pores fabricated at $15 \mathrm{~mW}$ and $1000 \mu \mathrm{ms}^{-1}$. B and $\mathrm{C}$ were imaged at $60^{\circ}$ tilt. Scale bar represents $1 \mu \mathrm{m}$ for all images. All samples were coated with a $10 \mathrm{~nm}$ gold layer prior to imaging.

The effect of fabrication parameters on pore array templates, and their subsequent impact on replica molded pillars has been documented hitherto. However, it is worthy of mention that the design of the fabrication file can also have a considerable impact on the fidelity of the negative structure, and the subsequent replica molded features. Figure 3 offers a direct comparison between design files when an additional outline is written at the perimeter of the cylindrical array. This outline, which is written prior to subsequent hatching, serves to greatly reduce the pore size achievable from the $800 \mathrm{~nm}$ features in the design file and limits the drastic effect of fabrication parameters on the resulting features. Figure $3 \mathrm{~A}$ shows a set of pores fabricated, in the absence of an additional contouring line, at $10 \mathrm{~mW}$ laser power and writing speeds from $1000-3000 \mu \mathrm{ms}^{-1}$ with top-down diameters measured from $1119 \pm 31 \mathrm{~nm}$ to $903 \pm 12$. The addition of a contouring line reduces this diameter, and further limits the effect of writing speed on the subsequent pores, with measured diameters of $528 \pm 21 \mathrm{~nm}$ and $557 \pm 21 \mathrm{~nm}$ for writing speeds of 1000 and 3000 $\mu \mathrm{ms}^{-1}$, respectively (Figure 3B). This had a profound effect on achieved diameters of replica molded pillars, which were measured from tilt SEM images to be $276 \pm 2 \mathrm{~nm}\left(1000 \mu \mathrm{ms}^{-1}\right)$ and $285 \pm 2 \mathrm{~nm}\left(3000 \mu \mathrm{ms}^{-1}\right)$, respectively. Most apparent, from Table S2 and Figure S4 is the narrow window of fabrication parameters which can be used to generate arrays that result in replica molding, when contour lines are employed. Further investigation is warranted to understand if this phenomenon is purely limited by pore dimensions, or if the variation of template modulus, caused by increasing levels of crosslinking along the contour line, is a contributing factor for replication of polymer structures on the nanoscale. 


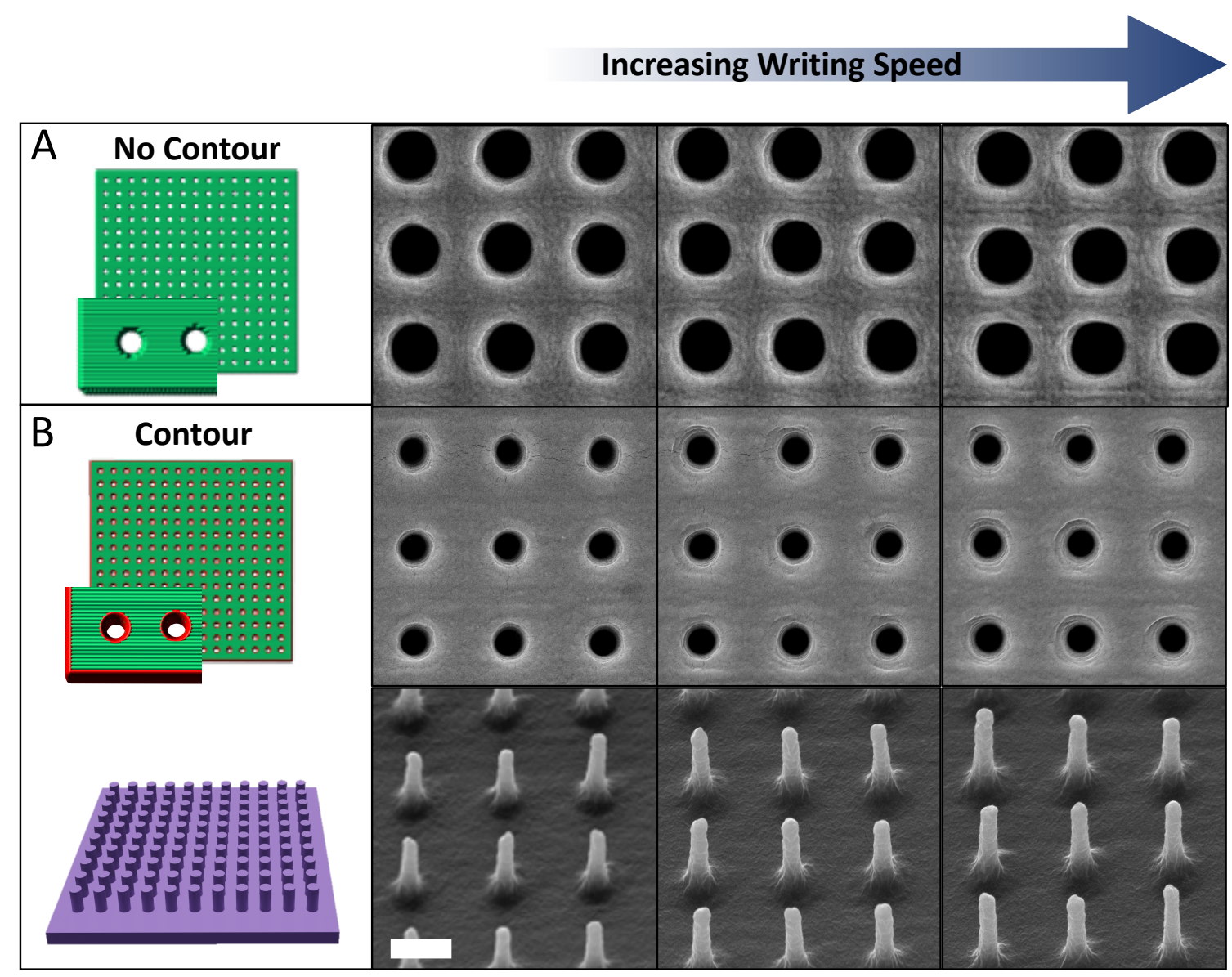

Figure 3: SEM images showing the effect of design on pore template and resulting replicated PEGDA pillars, when $10 \mathrm{~mW}$ laser power and writing speeds of $1000-3000 \mu \mathrm{ms}^{-1}$ are employed for master fabrication. A) in the absence of a contour line at the pore perimeter; B) with the inclusion of contour line for the same fabrication parameters (top); replica molded pillars in PEGDA at $45^{\circ}$ tilt (bottom). Scale bar represents $1 \mu \mathrm{m}$ for all images. All samples were coated with a $10 \mathrm{~nm}$ gold layer prior to imaging.

To truly exploit the potential of the methodologies presented herein, it is necessary to demonstrate the extension of this approach to larger-scale footprints, with retention of submicron resolution, homogeneity in heights, and applicability to a wider variety of materials. In this regard, 
the results presented in Figure 4 serve to address the extension of this approach to larger arrays. Accurate replication of a 900-pore array with the same feature dimension and pitch but a much larger footprint ( $50 \mu \mathrm{m} \times 50 \mu \mathrm{m}$; fabricated at $12.5 \mathrm{~mW}$ power and $\left.2000 \mu \mathrm{ms}^{-1}\right)$ is demonstrated in the SEM image in Figure 4A. Adaptation of the original stl design allows for fast and facile redesign of the template array. With the ability to seamlessly stitch $100 \mu \mathrm{m}$ x $100 \mu \mathrm{m}$ blocks, each with a fabrication time of a few minutes, the generation of a millimeter sized submicron structured array is easily achievable within a reasonable timeframe.

A

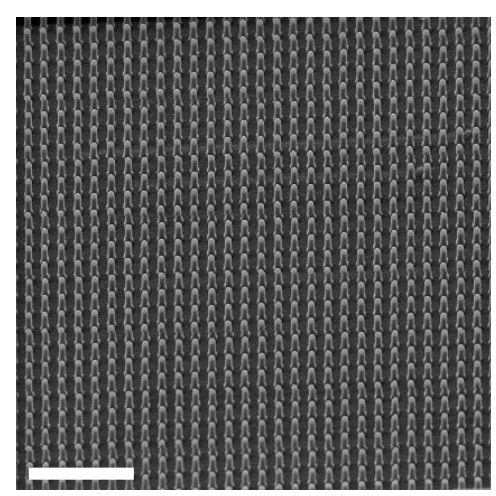

C

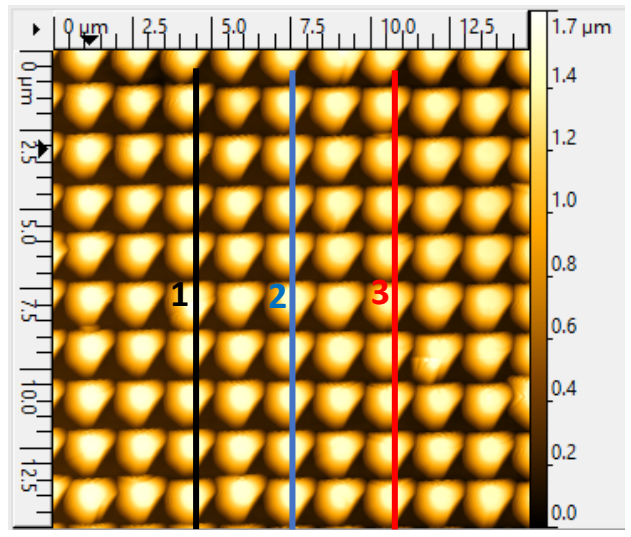

B

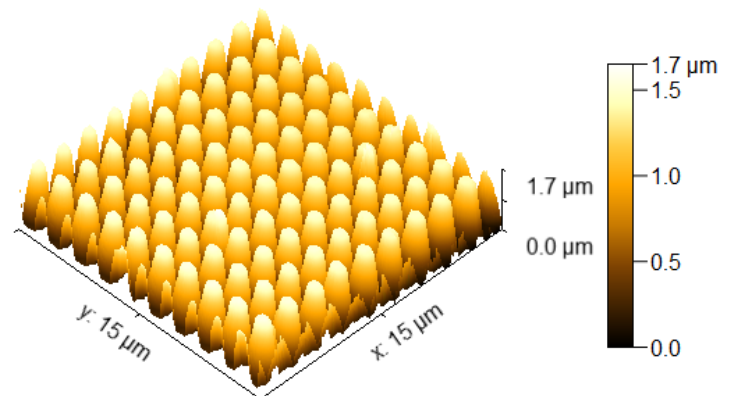

D

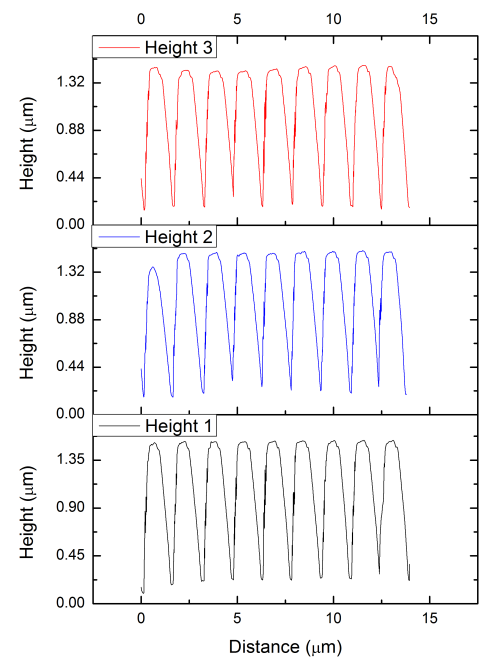

Figure 4: Production and characterization of larger scale replica molded arrays in PEGDA. A) SEM image of 30x30 replica molded PEGDA pillar array imaged at 55 tilt. Scale bar represents $10 \mu \mathrm{m}$. Sample was coated with a $10 \mathrm{~nm}$ gold layer prior to imaging; B) 3D reconstruction of AFM 
height analysis performed in tapping mode; C) and D) AFM height analysis and example of corresponding height profiles across the three traces shown.

Moreover, as demonstrated in Figure 4A, the significantly increased size of the array, and separation forces from $>600$ additional pillars posed no problem in replication of the master templated in PEGDA. Most importantly, AFM imaging of the PEGDA shows the homogeneity in height profile across a $15 \mu \mathrm{m} \times 15 \mu \mathrm{m}$ section, as seen in Figure 4B. Analysis of height traces, some examples of which are shown in in Figure 4C and Figure 4D, show an average height of $1440 \pm 43 \mathrm{~nm}(\mathrm{~N}=80$ pillars). Not only does this confirm such uniformity across the array, but it also serves to verify that infiltration of the prepolymer cocktail occurs to the very bottom of the $1.5 \mu \mathrm{m}$ cylindrical pores. After polymerization, complete removal of the newly formed pillars, with retention of the as-formed surface morphology, and minimal adherence or covalent attachment to the functionalized slide, offer much hope for the possibility of migrating to more compliant polymers.

Developments in 2PP for biological applications continue to make inroads in achieving $2 \mathrm{D}$ and 3D structures in biocompatible polymers. While some effective solutions to biocompatibility have been achieved through variation of photoinitiators, solvents, and suitable monomers, the nature of fabrication using DLW does necessitate a high degree of crosslinking, to enable small, selfstanding features. This can be achieved, as in the case of proprietary cocktails, through the use of multiarm acrylate monomers, or through the inclusion of additional crosslinking agents in bespoke prepolymer blends. The ability to accurately and predictably program the modulus of polymeric matrices is critical in biological applications, from gene therapy, to stem-cell differentiation. If successful, replica molding from high-resolution 2PP masters using traditional biocompatible prepolymer cocktails may serve to generate arrays of polymeric nanostructures with greater 
adaptability of physical and chemical properties, than can currently be achieved using $2 \mathrm{PP}$. To demonstrate the applicability and flexibility of this approach, a single $30 \times 30$ master array was fabricated without contour lines at $10 \mathrm{~mW}$ power and $1000 \mu \mathrm{ms}^{-1}$. From this same master, three cocktails were subsequently replica molded. The resulting pillar arrays are shown in Figure 5, and demonstrate complete replication across short-chain hydrophilic monomers, such as PEGDA $\left(\mathrm{M}_{\mathrm{n}}\right.$ 575) and PPGDA ( $\left.M_{n} 880\right)$, and the longer chain hydrophobic PCLDMA $\left(M_{n} 1350\right)$ monomer. Impressively, the retention of structure height $(1.5 \mu \mathrm{m})$ and width $(<500 \mathrm{~nm})$ in an elastomer of this nature, confirms the potential that this approach may hold in the generation of soft, submicron structured biocompatible and biodegradable polymers. 


\section{PP Master}

\section{PEGDA}

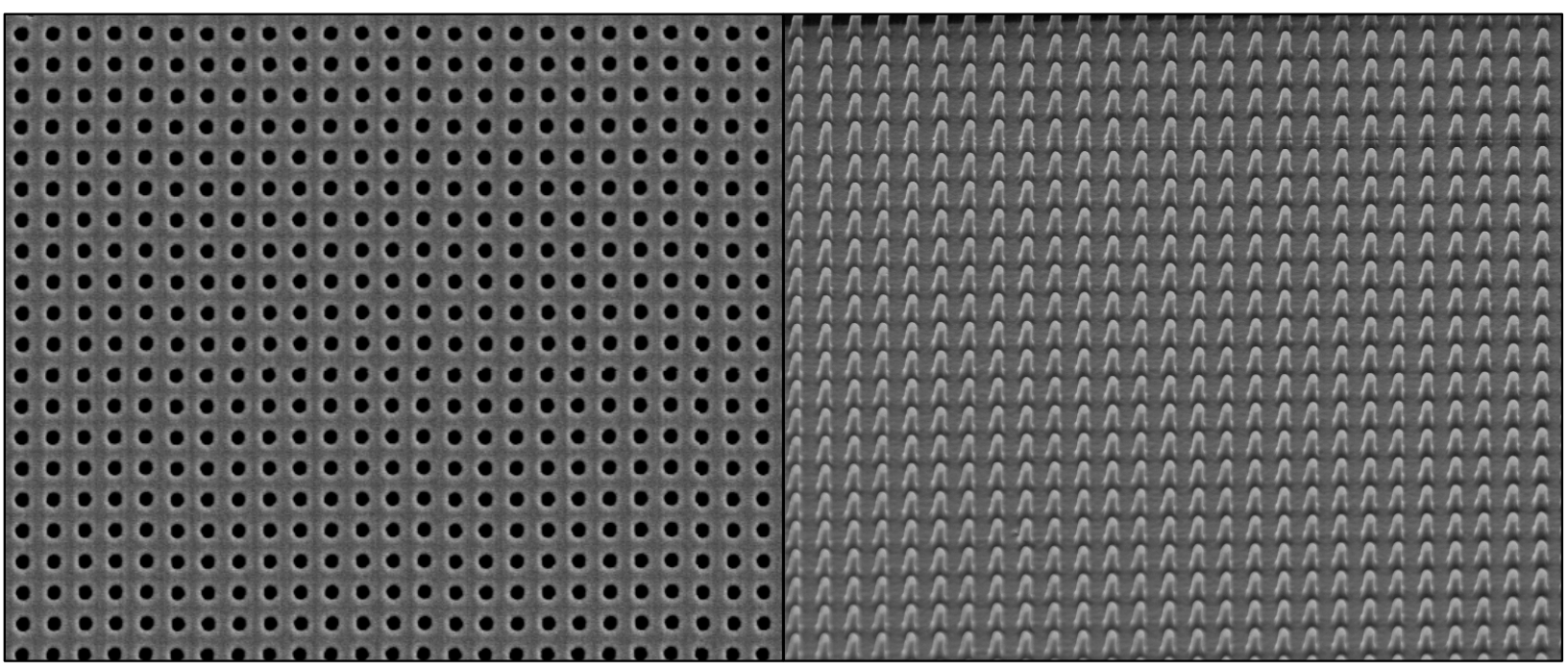

\section{PPGDA}

PCLDMA

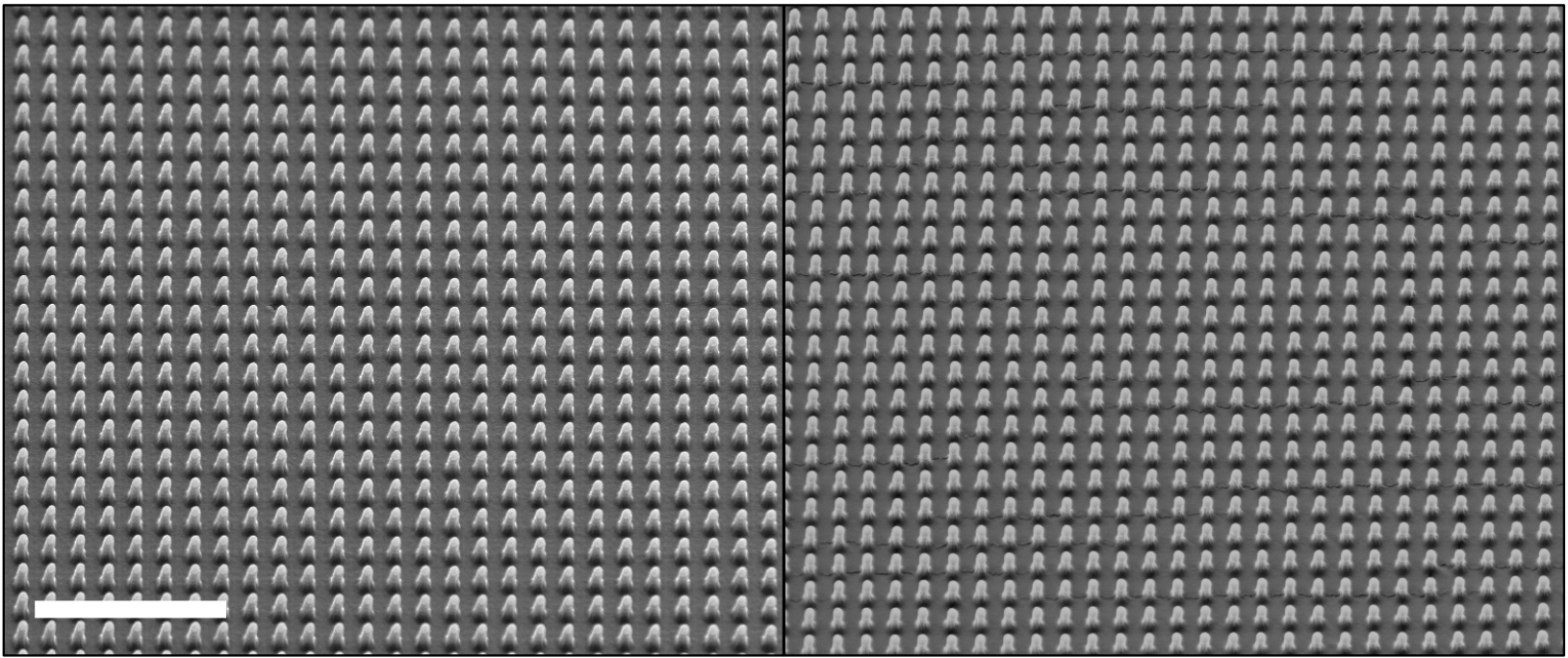

Figure 5: Multiple replications from a single 2PP master to produce submicron pillars in

poly(ethylene glycol) diacrylate $\left(\mathrm{M}_{\mathrm{n}}\right.$ 575) (PEGDA), poly(propylene glycol) diacrylate $\left(\mathrm{M}_{\mathrm{n}} 880\right)$

(PPGDA), and poly(caprolactone) dimethacrylate $\left(\mathrm{M}_{\mathrm{n}}\right.$ 1350) (PCLDMA). Replica molded arrays

are imaged at $45^{\circ}$ tilt. Scale bar for all samples represents $10 \mu \mathrm{m}$. All samples were coated with a

$10 \mathrm{~nm}$ gold layer prior to imaging. 
The mechanical properties of the nanostructured polymer surfaces were elucidated using AFM (see ESI) and compared to their 2PP fabricated analogues. Force volume mapping of the replicamolded samples demonstrated notably different stiffness regimes across the same sample. Applying masks allowed for analysis of three distinct regions on the nanostructured polymer surfaces; 1) bulk polymer; 2) pillar; and 3) flat regions at base of pillars. Images of modulus maps, masks used, and distribution of measurements can be found in Figures S5-S7. The bulk planar region of the replica molded PEGDA polymer exhibited a Young's modulus of $765 \pm 73 \mathrm{MPa}$, showing a considerable difference from the analogous PEGDA 2PP-fabricated sample, with a measured Young's modulus of $45 \pm 4 \mathrm{MPa}$. Such reduction in Young's modulus for 2PP-fabricated structures compared to bulk has been previously reported and is proposed to be a result of the different approaches used to induce polymerization. ${ }^{26,27}$ In the case of $2 \mathrm{PP}$, microstructures are constructed by building voxels on voxels, while bulk photoinitiated polymerization is achieved by light-flooding illumination. The higher molecular weight PPGDA gives a bulk polymer with a smaller Young's modulus of $374 \pm 41 \mathrm{MPa}$, as the flexibility of the chain increases.

The flat areas at the base of replica-molded pillars showed a noticeably lower Young's modulus for both polymers (405 $\pm 88 \mathrm{MPa}$ for PEGDA; $262 \pm 93 \mathrm{MPa}$ for PPGDA) than in the bulk regions. Of significant importance is the noticeable difference in Young's modulus measured for the replica molded pillars (111 MPa \pm 44 PEGDA, $76 \pm 22 \mathrm{MPa}$ for PPGDA) in comparison to the bulk regions of the respective polymers. This is best explained by the effect of polymerization in confined, submicron spaces. Although much of the previous studies on free radical polymerization in confined spaces focuses on linear polymer chains, ${ }^{28,29}$ the lower moduli of the submicron pillars could be a consequence of a lowered polymer glass transition temperature, caused by restriction of the polymeric network in a confined space. 


\section{Conclusions}

We have successfully created a highly adaptable and accessible means for low-cost generation of structured polymers, through the combination of $2 \mathrm{PP}$ and replica molding, which yields large arrays of nanostructures in soft, biocompatible polymers. Through optimization of 2PP design and fabrication parameters, we have demonstrated achievable control of feature size and shape of the negative 2PP master. Subsequent generation of highly reproducible pillared surfaces in photocurable polymers, such as PEGDA, PPGDA, and PCLDMA was then carried out, with size and morphology controlled by the master fabrication paraments. This strategy allows for the fabrication of desired 3D shapes in a variety of well-known, biocompatible materials with predefined and tailored modulus. The marriage of sub-micron resolution 3D fabrication with a wide library of materials of controlled mechanical properties, holds enormous potential for nextgeneration 3D cell assays. These large area, highly reproducible, structured polymers can be used to study invasion processes of cancer cells, or to probe the effect of the microenvironment on the differentiation of stem cells.

\section{Experimental}

Fabrication of Cylindrical Pore Arrays

Two photon polymerization of cylindrical pore arrays was carried out using a commercial direct laser writing apparatus, Photonic Professional (Nanoscribe Gmbh). The Dip-In configuration was used, employing a 63x objective. DiLL substrates and DiLL monomer photoresists were purchased from Nanoscribe. Prior to laser writing, the fused silica substrates $\left(25\right.$ x $25 \mathrm{~mm}^{2}$; thickness 0.7 $\mathrm{mm}$ ) were sonicated in ethanol for $30 \mathrm{~min}$, followed by UV Ozone treatment for $15 \mathrm{~min}$ to activate the substrate surface (Digital UV Ozone System, PSD Pro Series). The surface of the DiLL 
substrates was modified by immersion in a solution containing 3\% v/v 3-(trimethoxysilyl)propyl methacrylate (Sigma Aldrich) and $0.1 \% \mathrm{v} / \mathrm{v}$ acetic acid in ethanol for $1 \mathrm{hr}$. After rinsing, and drying under nitrogen, the substrates were cured for $10 \mathrm{mins}$ at $40{ }^{\circ} \mathrm{C}$. A single drop of the monomeric photoresist, IP-Dip (Nanoscribe Gmbh) was deposited onto the center of the substrate before the holder was placed into the system. The design (.stl) file of the desired array of cylindrical pores was loaded into the software with distances of $100 \mathrm{~nm}$ used for both slicing and hatching. For pore array fabrication, the laser power and scan speed were varied between $10-17.5 \mathrm{~mW}$ and $1000-$ $10000 \mu \mathrm{m} \mathrm{s}^{-1}$. After laser writing, the structures were cleaned by immersing in propylene glycol methyl ether acetate for $20 \mathrm{~min}$ followed by 2-propanol for $2 \mathrm{~min}$. The structures were then rinsed dried under a stream of nitrogen and coated with a $10 \mathrm{~nm}$ layer of gold.

Fabrication of Nanopillars

Replica molding from 2PP cylindrical pore arrays was carried out using a $1 \% \mathrm{w} / \mathrm{w}$ solution of phenylbis(2,4,6-trimethylbenzoyl)phosphine oxide in poly(ethylene glycol) diacrylate $\left(\mathrm{M}_{\mathrm{n}} 575\right)$ (Sigma Aldrich), a 1\% w/w solution of phenylbis(2,4,6-trimethylbenzoyl)phosphine oxide in poly(propylene glycol) diacrylate $\left(M_{n}\right.$ 880) (Sigma Aldrich), or a $2 \% \mathrm{w} / \mathrm{w}$ solution of phenylbis(2,4,6-trimethylbenzoyl)phosphine oxide in poly(caprolactone) dimethacrylate $\left(\mathrm{M}_{\mathrm{n}}\right.$ 1350) (Sigma Aldrich). Two PDMS spacers (thickness $600 \mu \mathrm{m}$ ) were placed firmly onto the Aucoated DiLL substrates and positioned either side of the 2PP structures. A cover glass (22 x 22 $\mathrm{mm}$ ) was placed firmly onto both PDMS spacers. The monomeric cocktail was introduced to the space beneath the sealed cover glass until the $2 \mathrm{PP}$ structures were fully covered. The cocktail was cured under white light (Edmund Optics, MI 150 Illuminator) for $120 \mathrm{~min}$.

SEM Imaging and Analysis 
Scanning Electron Microscopy of the 2PP cylindrical pore arrays and replica molded nanopillars was carried out using a Zeiss Ultra Plus Scanning Electron Microscope. An accelerating voltage of $5 \mathrm{kV}$ was used under SE2 mode to acquire all images. Prior to SEM imaging, the structures were coated with $10 \mathrm{~nm}$ Au-Pd layer using a Cressington Sputter Coater 208HR. A 57 x $0.1 \mathrm{~mm}$ Au-Pd target (TED PELLA INC.) was used to coat the structures under an inert atmosphere of Argon for $10 \mathrm{~s}$. Pore diameter was measured from top-down SEM images using the particle analysis function on ImageJ. A minimum of sixty pore diameter measurements were obtained per pore array fabricated to ensure a representative sample of the pores. The Feret diameter obtained for each pore was averaged and standard deviation obtained. The diameter of the nanopillars was obtained manually by measuring the width of the mid-section of the pillars using the measure tool.

\section{AFM Imaging and Analysis}

Atomic Force Microscopy of the 2PP cylindrical pore arrays and replica molded nanopillars was carried out using amplitude modulation AFM (Asylum Research, MFP3D) and a silicon probe (Budget Sensors, TAP300AI-G) with an aluminium reflective coating, a nominal resonance frequency of $300 \mathrm{kHz}$, and a nominal cantilever stiffness of $40 \mathrm{~N} / \mathrm{m}$. Prior to imaging, the substrate was secured onto a glass microscope slide. AFM images were post-processed and analyzed using Gwyddion. Line profile data was obtained following image post-processing and plotted in Origin. AFM force volume spectroscopy measurements were performed on the same system. IP-Dip samples were characterized using diamond probes (Adama, NM-RC) with a nominal cantilever stiffness of $350 \mathrm{~N} / \mathrm{m}$. For PEGDA and PPGDA samples, measurements were performed using $\mathrm{Si}$ probes (BudgetSensors, tip C, All-In-One-Al) with a nominal cantilever stiffness of $7.4 \mathrm{~N} / \mathrm{m}$. For 
all AFM force spectroscopy measurements, the cantilever spring constants were determined experimentally using the Sader method. ${ }^{30}$

Force volume mapping was implemented as a 100 x 100 array of force curves across a $2.5 \mu \mathrm{m}$ x $2.5 \mu \mathrm{m}$ area for IP-Dip samples (10,000 indentations per force map with a $25 \mathrm{~nm}$ spacing) and as a $150 \times 150$ array of force curves across a $2.5 \mu \mathrm{m} \times 2.5 \mu \mathrm{m}$ area for replica-molded samples (22,500 indentations per force map; $17 \mathrm{~nm}$ spacing). For PEGDA fabricated using 2PP, the force volume mapping was implemented as a 150 x 150 array across a $5 \mu \mathrm{m} \times 5 \mu \mathrm{m}(22,500$ indentations per force map; $33 \mathrm{~nm}$ spacing). A relative $5 \mu \mathrm{N}$ trigger force was used for IP-Dip samples, while a relative $250 \mathrm{nN}$ trigger force was used on PEGDA and PPGDA samples. The trigger forces were selected to ensure that indentations were limited to $10 \%$ of the sample thickness. ${ }^{31,32}$ Young's modulus was determined using the Asylum software by fitting the extension part of the forceindentation curves to the Hertz model ${ }^{33}$ using Poisson's ratios of 0.49 for IP-Dip samples and 0.35

for PEGDA and PPGDA samples. ${ }^{34,35}$

\section{ASSOCIATED CONTENT}

\section{Supporting Information}

The Supporting information is available free of charge at

SEM data, Replica molding results, AFM measurements. (PDF)

\section{AUTHOR INFORMATION}

\section{Corresponding Author}

E-mail: cdelane5@tcd.ie 


\section{Author Contributions}

The manuscript was written through contributions of all authors. All authors have given approval to the final version of the manuscript.

\section{ACKNOWLEDGMENT}

This work was funded by Science Foundation Ireland, through the Starting Investigator Research Grant (SIRG) Scheme (Grant Number 15/SIRG/3429). SK and NG also acknowledge SFI and the European Regional Development Fund (ERDF) (Grant Number 13/RC/2073 and 17/RCPhD/3480), through CURAM, the SFI Research Centre for Medical Devices. SK and MOL also acknowledge the Irish Research Council (IRC) (Grant number GOIPG/2017/1037). LF acknowledges the European Research Council (ERC) Starting Grant (project number 802929ChemLife) and SFI-ERDF (Grant Number 12/RC/2278_P2). The 2PP-DLW fabrication and SEM imaging for this project was carried out at the Additive Research Laboratory (AR-Lab) and the Advanced Microscopy Laboratory (AML), Trinity College Dublin, Ireland. The AR-Lab and AML are SFI supported centres, part of the CRANN Institute and affiliated to the AMBER centre.

\section{REFERENCES}

(1) Zhang, S.; Chen, Y. Nanofabrication and Coloration Study of Artificial Morpho Butterfly Wings with Aligned Lamellae Layers. Sci. Rep. 2015, 5 (1), 16637. https://doi.org/10.1038/srep16637.

(2) Narasimhan, V.; Siddique, R. H.; Lee, J. O.; Kumar, S.; Ndjamen, B.; Du, J.; Hong, N.; Sretavan, D.; Choo, H. Multifunctional Biophotonic Nanostructures Inspired by the Longtail Glasswing Butterfly for Medical Devices. Nat. Nanotechnol. 2018, 13 (6), 512519. https://doi.org/10.1038/s41565-018-0111-5. 
(3) Hsiung, B. K.; Siddique, R. H.; Stavenga, D. G.; Otto, J. C.; Allen, M. C.; Liu, Y.; Lu, Y. F.; Deheyn, D. D.; Shawkey, M. D.; Blackledge, T. A. Rainbow Peacock Spiders Inspire Miniature Super-Iridescent Optics. Nat. Commun. 2017, $8 \quad$ (1), 1-8. https://doi.org/10.1038/s41467-017-02451-x.

(4) Zyla, G.; Kovalev, A.; Grafen, M.; Gurevich, E. L.; Esen, C.; Ostendorf, A.; Gorb, S. Generation of Bioinspired Structural Colors via Two-Photon Polymerization. Sci. Rep. 2017, 7 (1), 1-9. https://doi.org/10.1038/s41598-017-17914-w.

(5) Kelleher, S. M.; Habimana, O.; Lawler, J.; O’ Reilly, B.; Daniels, S.; Casey, E.; Cowley, A. Cicada Wing Surface Topography: An Investigation into the Bactericidal Properties of Nanostructural Features. ACS Appl. Mater. Interfaces 2016, 8 (24), 14966-14974. https://doi.org/10.1021/acsami.5b08309.

(6) Watson, G. S.; Green, D. W.; Cribb, B. W.; Brown, C. L.; Meritt, C. R.; Tobin, M. J.; Vongsvivut, J.; Sun, M.; Liang, A.-P.; Watson, J. A. Insect Analogue to the Lotus Leaf: A Planthopper Wing Membrane Incorporating a Low-Adhesion, Nonwetting, Superhydrophobic, Bactericidal, and Biocompatible Surface. ACS Appl. Mater. Interfaces 2017, 9 (28), 24381-24392. https://doi.org/10.1021/acsami.7b08368.

(7) Wi, J. S.; Lee, H. S.; Lim, K.; Nam, S. W.; Kim, H. M.; Park, S. Y.; Lee, J. J.; Hong, C. D.; Jin, S.; Kim, K. B. Fabrication of Silicon Nanopillar Teradot Arrays by Electron-Beam Patterning for Nanoimprint Molds. Small 2008, 4 (12), 2118-2122. https://doi.org/10.1002/smll.200800625.

(8) Lipomi, D. J.; Martinez, R. V.; Cademartiri, L.; Whitesides, G. M. Soft Lithographic Approaches to Nanofabrication. Polym. Sci. A Compr. Ref. 10 Vol. Set 2012, 7, 211-231. 
https://doi.org/10.1016/B978-0-444-53349-4.00180-1.

(9) Acikgoz, C.; Hempenius, M. A.; Huskens, J.; Vancso, G. J. Polymers in Conventional and Alternative Lithography for the Fabrication of Nanostructures. Eur. Polym. J. 2011, 47 (11), 2033-2052. https://doi.org/10.1016/j.eurpolymj.2011.07.025.

(10) Mijangos, C.; Hernández, R.; Martín, J. A Review on the Progress of Polymer Nanostructures with Modulated Morphologies and Properties, Using Nanoporous AAO Templates. Prog. Polym. Sci. 2016, 54-55, 148-182. https://doi.org/10.1016/j.progpolymsci.2015.10.003.

(11) Varapnickas, S.; Malinauskas, M. Processes of Direct Laser Writing 3D Nano-Lithography. Preprints 2018, 2018120119, 1-31. https://doi.org/10.20944/preprints201812.0119.v1.

(12) Sun, H. B.; Maeda, M.; Takada, K.; Chon, J. W. M.; Gu, M.; Kawata, S. Experimental Investigation of Single Voxels for Laser Nanofabrication via Two-Photon Photopolymerization. Appl. Phys. Lett. 2003, $83 \quad$ (5), 819-821. https://doi.org/10.1063/1.1598293.

(13) Haske, W.; Chen, V. W.; Hales, J. M.; Dong, W.; Barlow, S.; Marder, S. R.; Perry, J. W. $65 \mathrm{Nm}$ Feature Sizes Using Visible Wavelength 3-D Multiphoton Lithography. Opt. Express 2007, 15 (6), 3426. https://doi.org/10.1364/oe.15.003426.

(14) Accardo, A.; Blatché, M. C.; Courson, R.; Loubinoux, I.; Vieu, C.; Malaquin, L. TwoPhoton Lithography and Microscopy of 3D Hydrogel Scaffolds for Neuronal Cell Growth. Biomed. Phys. Eng. Express 2018, 4 (2). https://doi.org/10.1088/2057-1976/aaab93.

(15) LaFratta, C.; Baldacchini, T. Two-Photon Polymerization Metrology: Characterization 
Methods of Mechanisms and Microstructures. Micromachines 2017, 8 (4), 101. https://doi.org/10.3390/mi8040101.

(16) Purtov, J.; Verch, A.; Rogin, P.; Hensel, R. Improved Development Procedure to Enhance the Stability of Microstructures Created by Two-Photon Polymerization. Microelectron. Eng. 2018, 194 (December 2017), 45-50. https://doi.org/10.1016/j.mee.2018.03.009.

(17) Purtov, J.; Rogin, P.; Verch, A.; Johansen, V. E.; Hensel, R. Nanopillar Diffraction Gratings by Two-Photon Lithography. Nanomaterials 2019, 9, 1495.

(18) Nguyen, A. K.; Narayan, R. J. Two-Photon Polymerization for Biological Applications. Mater. Today 2017, 20 (6), 314-322. https://doi.org/10.1016/j.mattod.2017.06.004.

(19) Nazir, R.; Danilevicius, P.; Ciuciu, A. I.; Chatzinikolaidou, M.; Gray, D.; Flamigni, L.; Farsari, M.; Gryko, D. T. П-Expanded Ketocoumarins As Efficient, Biocompatible Initiators for Two-Photon-Induced Polymerization. Chem. Mater. 2014, 26 (10), 31753184. https://doi.org/10.1021/cm500612w.

(20) Nguyen, A. K.; Gittard, S. D.; Koroleva, A.; Schlie, S.; Gaidukeviciute, A.; Chichkov, B. N.; Narayan, R. J. Two-Photon Polymerization of Polyethylene Glycol Diacrylate Scaffolds with Riboflavin and Triethanolamine Used as a Water-Soluble Photoinitiator. Regen. Med. 2013, 8 (6), 725-738. https://doi.org/10.2217/rme.13.60.

(21) Jhaveri, S. J.; McMullen, J. D.; Sijbesma, R.; Tan, L.-S.; Zipfel, W.; Ober, C. K. Direct Three-Dimensional Microfabrication of Hydrogels via Two-Photon Lithography in Aqueous Solution. Chem. Mater. 2009, $21 \quad$ (10), 2003-2006. https://doi.org/10.1021/cm803174e. 
(22) Cadarso, V. J.; Chidambaram, N.; Jacot-Descombes, L.; Schift, H. High-Aspect-Ratio Nanoimprint Process Chains. Microsystems Nanoeng. 2017, 3 (September 2016), 1-12. https://doi.org/10.1038/micronano.2017.17.

(23) LaFratta, C. N.; Baldacchini, T.; Farrer, R. A.; Fourkas, J. T.; Teich, M. C.; Saleh, B. E. A.; Naughton, M. J. Replication of Two-Photon-Polymerized Structures with Extremely High Aspect Ratios and Large Overhangs. J. Phys. Chem. B 2004, 108 (31), 11256-11258. https://doi.org/10.1021/jp048525r.

(24) Sun, X.; Hourwitz, M. J.; Baker, E. M.; Schmidt, B. U. S.; Losert, W.; Fourkas, J. T. Replication of Biocompatible, Nanotopographic Surfaces. Sci. Rep. 2018, 8 (1), 1-9. https://doi.org/10.1038/s41598-017-19008-z.

(25) LaFratta, C. M.; Li, L.; Fourkas, J. T. Soft-Lithographic Replication of 3D Microstructures with Closed Loops. Proc. Natl. Acad. Sci. U. S. A. 2006, 103 (23), 8589-8594. https://doi.org/10.1073/pnas.0603247103.

(26) Nakanishi, S.; Shoji, S.; Kawata, S.; Sun, H. B. Giant Elasticity of Photopolymer Nanowires. Appl. Phys. Lett. 2007, 91 (6), 2005-2008. https://doi.org/10.1063/1.2767995.

(27) Cadarso, V. J.; Chidambaram, N.; Jacot-Descombes, L.; Schift, H. High-Aspect-Ratio Nanoimprint Process Chains. Microsystems Nanoeng. 2017, 3 (September 2016), 1-12. https://doi.org/10.1038/micronano.2017.17.

(28) Sanz, B.; Ballard, N.; M. Asua, J.; Mijangos, C. Effect of Confinement on the Synthesis of PMMA in AAO Templates and Modeling of Free Radical Polymerization. Macromolecules 2017, 50 (3), 811-821. https://doi.org/10.1021/acs.macromol.6b02282. 
(29) Tarnacka, M.; Maksym, P.; Zięba, A.; Mielańczyk, A.; Geppert-Rybczyńska, M.; LeonBoigues, L.; Mijangos, C.; Kamiński, K.; Paluch, M. The Application of Spatially Restricted Geometries as a Unique Route to Produce Well-Defined Poly(Vinyl Pyrrolidones) via Free Radical Polymerisation. Chem. Commun. 2019, 55 (45), 6441-6444. https://doi.org/10.1039/C9CC02625H.

(30) Sader, J. E.; Chon, J. W. M.; Mulvaney, P. Calibration of Rectangular Atomic Force Microscope Cantilevers. Rev. Sci. Instrum. 1999, 70 (10), 3967-3969. https://doi.org/10.1063/1.1150021.

(31) Costa, K. D.; Yin, F. C. P. Analysis of Indentation: Implications for Measuring Mechanical Properties with Atomic Force Microscopy. J. Biomech. Eng. 1999, 121 (5), 462-471. https://doi.org/10.1115/1.2835074.

(32) Wojcikiewicz, E.P.; Zhang, X.; Moy, V. T. Force and Compliance Measurements on Living Cells Using Atomic Force Microscopy (AFM). Biol. Proced. Online 2004, 6 (1), 1-9. https://doi.org/10.1251/bpo67.

(33) Dimitriadis, E. K.; Horkay, F.; Maresca, J.; Kachar, B.; Chadwick, R. S. Determination of Elastic Moduli of Thin Layers of Soft Material Using the Atomic Force Microscope. Biophys. J. 2002, 82 (5), 2798-2810. https://doi.org/10.1016/S0006-3495(02)75620-8.

(34) Greaves, G. N.; Greer, A. L.; Lakes, R. S.; Rouxel, T. Poisson's Ratio and Modern Materials. Nat. Mater. 2011, 10 (11), 823-837. https://doi.org/10.1038/nmat3134.

(35) Lemma, E. D.; Rizzi, F.; Sileo, L.; Spagnolo, B.; Dattoma, T.; Qualtieri, A.; Vittorio, M. De; Pisanello, F. Static and Dynamic Mechanical Characterization of Two-Photon 
Lithography Photoresists. 2015, 2-3. 


\section{TOC ENTRY}

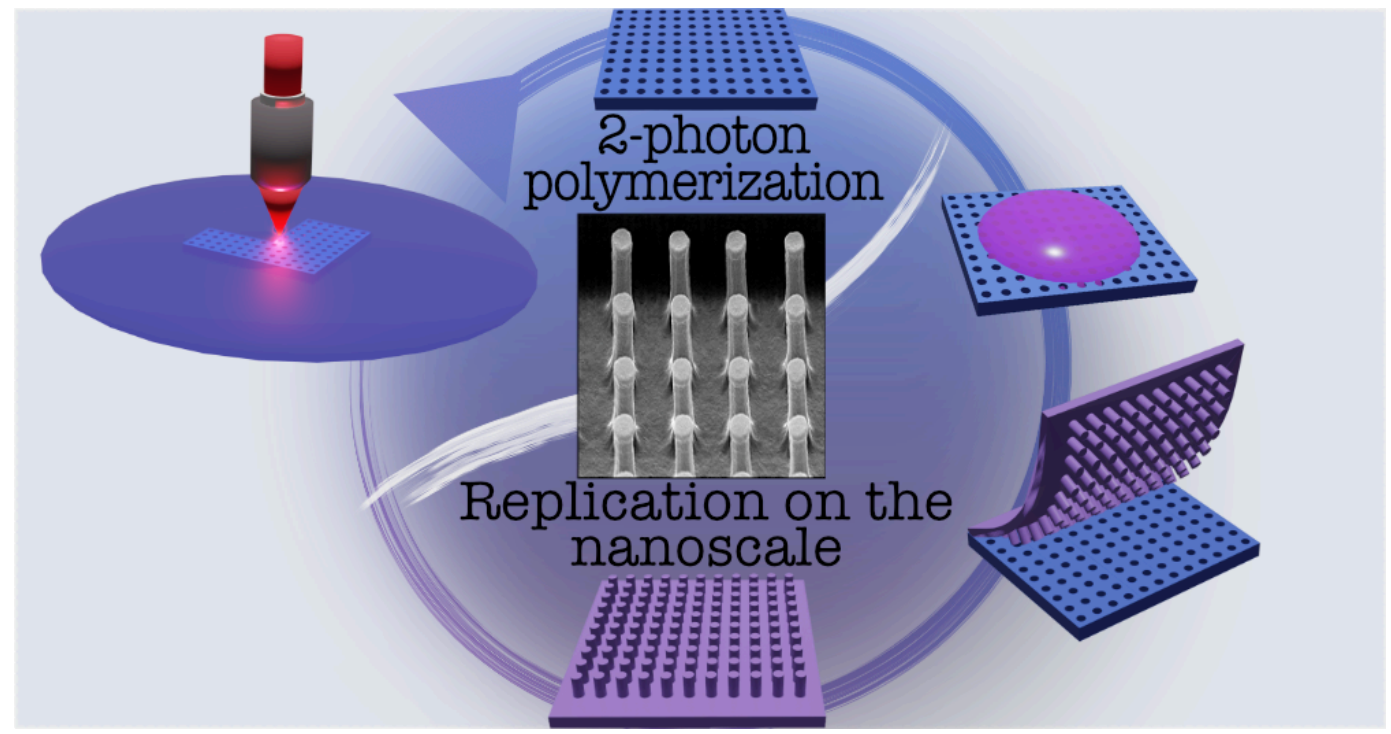

Direct Laser writing is used as a means to generate easily adapted templates for replica molding polymeric nanopillars. Optimization of fabrication and design parameters show the significant impact on pore arrays and the resulting nanopillars. Variation of monomeric cocktails shows the ability to extend the methodology to nanostructure soft, biocompatible, biodegradable polymers. 